\title{
Réseaux de collecte et de distribution du lait dans les villes d'Afrique soudano-sahélienne
}

\author{
C. Corniaux ${ }^{1 *}$ B. Bonfoh ${ }^{2}$ A. Diallo ${ }^{3}$ \\ R. Poccard-Chapuis ${ }^{4}$ G. Vias ${ }^{5}$
}

\section{Mots-clés}

Collecte de lait - Circuit de commercialisation - Analyse de réseau - Environnement urbain Afrique.

\begin{abstract}
Résumé
Dans les villes secondaires d'Afrique soudano-sahélienne, la proximité du milieu rural permet encore la collecte du lait auprès des producteurs situés en zone périurbaine et sa distribution auprès des consommateurs urbains. De nombreux acteurs transportent et commercialisent de petites quantités. Des minilaiteries prennent parfois une place majeure en tant que relais. En revanche, le fossé se creuse dans les capitales tant que le réseau de collecte et de distribution ne s'adapte pas aux exigences du marché. Les minilaiteries, en dépit de leur dynamisme, ne répondent que très partiellement à cette nécessité d'adaptation. La croissance démographique, I'expansion urbaine en périphérie au détriment des pâturages ou encore l'éloignement progressif de la production par rapport aux lieux de consommation rendent caduques les systèmes traditionnels de transport. Pour que la production laitière locale garde une place significative sur un marché urbain porteur, la collecte " industrielle » dans les lieux de production devrait être promue en dépit de leur éloignement, à l'instar de la laiterie Tiviski en Mauritanie. Mais les conditions de l'industrialisation ne sont pas évidentes à réunir. En outre, les réseaux de distribution du lait en poudre importé et de ses produits dérivés suivent efficacement les transformations récentes des marchés urbains (multiplication des boutiquiers, diversification des produits, présence croissante de la chaîne du froid, prix attractifs, qualité sanitaire correcte...), rendant encore plus rude, voire illusoire la compétition. Les politiques publiques doivent tenir compte de cet état de fait. L'appui aux unités de collecte et de distribution du lait local est une priorité, sous réserve que le développement de la production locale en soit une aux yeux des dirigeants.
\end{abstract}

\section{INTRODUCTION}

En Afrique subsaharienne, le développement des villes rend de plus en plus difficile leur approvisionnement en produits laitiers et la demande urbaine est en hausse constante (11). Dans les pays sahéliens (Burkina Faso, Mali, Mauritanie, Niger, Sénégal), cette demande accrue s'est traduite par une augmentation des importations.

\footnotetext{
1. IER, BP 1813, Bamako, Mali ; Cirad, UPR Systèmes d'élevage, campus de Baillarguet, TAC-18/A, F-34398 Montpellier, France.

E-mail : christian.corniaux@cirad.fr

2. Institut du Sahel, Bamako, Mali.

3. ICD, Bamako, Mali.

4. IER, Sikasso, Mali ; Cirad, UPR Systèmes d'élevage, F-34398 Montpellier, France.

5. Karkara, Niamey, Niger.

* Auteur pour la correspondance
}

Alors que ces pays disposent d'un important élevage pastoral et agropastoral, les importations de lait sont devenues structurelles et leur balance commerciale est devenue très largement déficitaire, malgré la dévaluation du franc CFA intervenue en 1994 (11, 12).

Pour résoudre ce déficit, les réponses des autorités publiques, relayées par les services en charge de la filière, sont généralement de deux ordres : il s'agit d'une part d'intensifier la production, notamment avec des programmes de sédentarisation des troupeaux laitiers et d'insémination artificielle et, d'autre part, de taxer les importations. Pourtant, à supposer que ces politiques soient applicables et efficaces, une question demeure : comment mettre en adéquation une offre potentielle de lait et la demande urbaine ? Les réseaux de collecte et de distribution sont au cœur de ce questionnement. Le transport est un élément majeur dans l'organisation des flux, d'autant plus que le lait frais est un produit hautement périssable dans les conditions soudano-sahéliennes. Plusieurs travaux 
soulignent le rôle de l'organisation des systèmes commerciaux dans l'essor de la production laitière locale $(3,7)$. La présente analyse a eu pour objectif de faire le point des connaissances sur ces systèmes. L'étude se base sur la synthèse de travaux menés dans différents pays d'Afrique de l'Ouest. Après avoir proposé un diagnostic de la situation passée et actuelle dans les capitales et les villes secondaires de la sous-région, les atouts et les faiblesses de ces réseaux de collecte et de distribution du lait local ont été analysés. Enfin, ces systèmes de commercialisation du lait local ont été confrontés à ceux du lait importé afin d'en retirer des enseignements susceptibles d'orienter les politiques publiques.

\section{METHODES}

Cette étude a été basée sur une revue bibliographique disponible dans les pays de la sous-région. Elle porte notamment sur l'évaluation des niveaux de couverture des besoins des principales villes d'Afrique soudano-sahélienne $(1,3,4,5,6,11)$. La revue a été complétée par une enquête de terrain réalisée en 2006 dans cinq villes maliennes : Bamako, Koutiala, Mopti, Ségou et Sikasso. Ces villes se distinguaient par leur taille et leurs réseaux de collecte. Deux laiteries industrielles et des minilaiteries étaient présentes à Bamako. Des minilaiteries s'étaient développées à Koutiala et Mopti.

Pour cette enquête, la population impliquée dans le commerce urbain des produits laitiers a été dénombrée dans chaque quartier des villes étudiées. Un comptage exhaustif des acteurs de la commercialisation a été réalisé, sauf pour les boutiquiers de Bamako qui ont fait l'objet d'une estimation. Sur la base d'un échantillonnage aléatoire de 1 à 5 acteurs par quartier, des entretiens ont été effectués auprès de 702 personnes, en un seul passage (tableau I). Lorsque le nombre d'acteurs par zone dépassait 100 individus, les auteurs se fixaient un seuil minimum de 30 questionnaires. Lorsque ce nombre était inférieur à 100 individus, un seuil minimum de 30 p. 100 d'acteurs à enquêter était fixé. Les questions ont porté sur les types de produits et les quantités collectées ou distribuées par les différents groupes d'acteurs. Chaque acteur a été localisé, puis questionné sur la qualité des produits [type de produits laitiers (lait frais ou caillé, yaourt, crème maturée, beurre liquide, poudre de lait, lait condensé), marque quand produit transformé, conditionnement (louche, sachet, en vrac, boîte), prix] et sur les quantités commercialisées (volume, période de vente). La compilation des résultats a permis d'élaborer des statistiques sur la distribution du lait par catégorie de produits selon les acteurs et la ville considérée.

\section{RESULTATS}

\section{Niveau de couverture des besoins en produits laitiers dans plusieurs villes d'Afrique soudano-sahélienne}

Afin de juger de l'efficacité actuelle des réseaux de collecte, il était indispensable de connaître la part du lait produit localement dans la couverture des besoins des populations urbaines en produits laitiers. En dépit de leur importance stratégique, il existe peu de données de ce type dans la littérature. Elles sont en outre souvent partielles et parfois sujettes à caution.

La situation est apparue contrastée entre les grandes villes et les villes secondaires de moins de 150000 habitants. Dans les grands centres urbains, la couverture locale en produits laitiers n'a pas généralement dépassé le seuil de 10 p. 100 * et a même été de l'ordre de 5 p. 100 dans les grandes capitales (Bamako, Dakar). A contrario, la couverture locale a été plus importante dans les villes secondaires puisqu'elle a été au minimum de 10 p. 100, pouvant atteindre la moitié des besoins (cas de Mopti et Niono) (tableaux II et III). Par ailleurs, sur la base d'enquêtes déclaratives de consommation, Poccard-Chapuis et coll. (13) estiment la part du lait local dans la consommation à 50 p. 100 à Ségou et à Sikasso. Cette part est sans doute surévaluée (enquête en un seul passage), mais ces résultats soulignent l'importance des produits laitiers locaux dans les villes secondaires.

Dans ce contexte, deux villes présentaient une singularité : Nouakchott (Mauritanie) et Saint-Louis (Sénégal). Ainsi, Nouakchott a eu un taux de couverture nettement supérieur à celui des capitales voisines (15 à 20 p. 100 vs moins de 10 p. 100). Certes la taille de sa population était plus modeste et les Mauritaniens appréciaient le lait frais. Mais la différence majeure tenait dans l'approvisionnement de Nouakchott assuré par des laiteries qui collectaient dans la vallée du fleuve Sénégal, lieu de production situé à $250 \mathrm{~km}$ au sud.

\footnotetext{
* A Niamey, les fourchettes étaient trop larges pour se prononcer clairement et les importations étaient relativement faibles au Niger comparativement aux autres pays sahéliens.
}

Tableau I

Nombre d'acteurs intérrogés par type et par ville

\begin{tabular}{|c|c|c|c|c|c|}
\hline Type d'acteur & Bamako & Koutiala & Mopti & Ségou & Sikasso \\
\hline Boutiquier & $41(3800) *$ & $41(210)$ & $26(380)$ & $33(350)$ & $36(630)$ \\
\hline Etalagiste & - & - & - & $18(250)$ & $22(250)$ \\
\hline Cafetier & $33(270)$ & $28(90)$ & $11(50)$ & $28(70)$ & $26(70)$ \\
\hline Vendeuse ambulante & $31(125)$ & $17(20)$ & $12(60)$ & $36(50)$ & $0(50)$ \\
\hline Vendeuse fixe & $19(115)$ & $13(35)$ & $22(50)$ & $23(50)$ & $24(50)$ \\
\hline Transformatrice & $34(180)$ & $0(?)$ & $8(8)$ & $5(7)$ & $24(24)$ \\
\hline Cycliste/moto & $30(?)$ & $0(?)$ & $15(20)$ & $15(35)$ & $0(40)$ \\
\hline Grossiste & - & $8(10)$ & $5(5)$ & $10(11)$ & - \\
\hline Minilaiterie & $4(10)$ & $2(2)$ & $1(1)$ & $0(0)$ & $1(1)$ \\
\hline Laiterie & $2(2)$ & $0(0)$ & $0(0)$ & $0(0)$ & $0(0)$ \\
\hline
\end{tabular}

* Entre parenthèses : estimation du nombre d'acteurs par catégorie et par ville enquêtée 
En revanche, Saint-Louis, ville de 175000 habitants, a eu un taux de couverture faible, de l'ordre de 5 p. 100. Les quantités livrées quotidiennement étaient pourtant relativement importantes comparées à celles des autres villes secondaires (tableau III). En fait, cette ville se singularise surtout par sa demande urbaine : SaintLouis consomme beaucoup de lait que son élevage périurbain n'est pas aujourd'hui en mesure de satisfaire. La mise en place en 2006 de la Laiterie du berger à Richard-Toll (située à 100 km de Saint-Louis, considérée comme son marché potentiel) pourrait néanmoins faire évoluer la couverture locale de Saint-Louis. D'une collecte journalière initiale de $650 \mathrm{~L}$ /jour, le volume a atteint 2000 L/jour dès l'hivernage 2007. Cette production est cependant destinée en priorité à Dakar.

Pour autant, quelle qu'ait été la ville considérée, à l'exception notable de Nouakchott, l'approvisionnement local issu de la production périurbaine est resté modeste en volume (évaluée à moins de $10000 \mathrm{~L}$ par jour). Tant que la taille de la ville et sa demande en produits laitiers demeuraient réduites, situation des villes secondaires, le taux de couverture pouvait dépasser 10 p. 100 et même atteindre 50 p. 100. En revanche, quand la demande augmentait significativement (croissance démographique ou augmentation de la consommation individuelle), les systèmes de collecte en place ne semblaient plus en mesure de s'adapter pour y répondre. Comment peut-on expliquer ce défaut de réactivité des systèmes de collecte locale ? Pour répondre à cette question, il est utile de décrire les acteurs en charge de cette collecte.

\section{Principaux acteurs de la collecte et de la distribution au détail des produits laitiers : le cas du Mali}

Au Mali, les acteurs de la collecte, de la transformation et de la distribution des produits laitiers locaux et importés étaient nombreux.

Les collecteurs se chargeaient de l'achat du lait frais chez les producteurs d'une concession ou d'un village pour le convoyer auprès de vendeuses ou de laiteries. C'étaient le plus souvent des hommes qui se déplaçaient en vélo ou en moto. Le transport se faisait dans des bidons. Parfois c'étaient des femmes qui achetaient du lait (frais ou caillé) sur les marchés ruraux pour le revendre en ville. Elles utilisaient les transports en commun.

\section{Tableau II}

Estimation de la demande urbaine en produits laitiers * et de la couverture locale dans plusieurs villes d'Afrique de l'Ouest soudano-sahélienne

\begin{tabular}{|c|c|c|c|c|c|c|c|c|}
\hline & \multicolumn{5}{|c|}{1995 (Corniaux, 2003) } & \multicolumn{3}{|c|}{2006 ** } \\
\hline & \multirow[t]{2}{*}{ Population } & \multirow{2}{*}{$\begin{array}{c}\text { Demande } \\
\text { urbaine } \\
(\mathrm{L} / \mathrm{j})\end{array}$} & \multicolumn{2}{|c|}{$\begin{array}{c}\text { Couverture } \\
\text { locale }\end{array}$} & \multirow[t]{2}{*}{$\begin{array}{l}\text { Population } \\
\text { (millions) }\end{array}$} & \multirow{2}{*}{$\begin{array}{c}\text { Demande } \\
\text { urbaine } \\
(\mathrm{L} / \mathrm{j})\end{array}$} & \multicolumn{2}{|c|}{$\begin{array}{c}\text { Couverture } \\
\text { locale }\end{array}$} \\
\hline & & & $(\mathrm{L} / \mathrm{j})$ & $(\%)$ & & & $(\mathrm{L} / \mathrm{j})$ & $(\%)$ \\
\hline Bamako & 1,0 & 90000 & 8000 & 8 & 1,2 & $100000-130000$ & $4000-7000$ & $4-6$ \\
\hline Bobo-Dioulasso & 0,3 & 18000 & 1750 & 9 & 0,6 & $15000-25000$ & $1500-2500$ & $8-12$ \\
\hline Dakar & 1,7 & 150000 & 3000 & 2 & 2,0 & $210000-230000$ & $10000-13000$ & $4-5$ \\
\hline Niamey & - & - & - & - & 0,9 & $70000-100000$ & $7000-20000$ & $7-25$ \\
\hline Nouakchott & - & - & - & - & 0,6 & $100000-150000$ & $20000-25000$ & $15-20$ \\
\hline
\end{tabular}

* Hors beurre, sirimé, fromage et crème

** Pour Bamako : d'après les enquêtes de l'étude ; pour Bobo-Dioulasso (Burkina Faso) : d'après Hamadou (commun. pers., 2007) ; pour Dakar : d'après Desmoulin, 2006 ; pour Niamey : d'après S. Boubacar (Solani) (commun. pers., 2007); pour Nouakchott : d'après données 2006 de collecte de l'entreprise Tiviski

\section{Tableau III}

Estimation de la demande urbaine en produits laitiers * et de la couverture locale dans certaines villes secondaires d'Afrique de l'Ouest soudano-sahélienne

$\begin{array}{cccc}\text { Population } & \begin{array}{c}\text { Demande urbaine } \\ (\mathrm{L} / \mathrm{j})\end{array} & \begin{array}{c}\text { Couverture locale } \\ (\mathrm{L} / \mathrm{j})\end{array} & \text { Références }\end{array}$

\section{Sénégal}

$\begin{array}{lccccc}\begin{array}{l}\text { Saint-Louis } \\ \text { Kolda }\end{array} & 175000 & 45000-55000 & 2000-3000 & 4-6 & \begin{array}{l}\text { (Corniaux, 2005) } \\ \text { (Dieye, 2006) }\end{array} \\ \begin{array}{l}\text { Mali } \\ \text { Mopti }\end{array} & & & 1000-1500 & & \\ \text { Sikasso } & 95000 & 8000-12000 & 4000-5000 & 40-55 & \text { Enquêtes de l'étude } \\ \text { Ségou } & 120000 & 10000-15000 & 1000-2500 & 10-20 & \text { Enquêtes de l'étude } \\ \text { Niono } & 100000 & 9000-13000 & 1000-2500 & 10-25 & \text { Enquêtes de l'étude } \\ \text { (Duteurtre, 2000) }\end{array}$

* Hors beurre, sirimé, fromage et crème 
Les vendeuses ambulantes étaient des femmes d'éleveurs qui vendaient directement leur lait ou celui de leur famille. Elles se déplaçaient à pied en ville en faisant du porte à porte, la calebasse sur la tête. Elles proposaient du lait frais ou du lait caillé (accompagné de couscous de mil ou de riz).

Les vendeuses fixes étaient généralement des femmes d'éleveurs ou de particuliers qui s'installaient au niveau des marchés urbains. Elles commercialisaient du lait frais, du lait caillé, parfois du féné (crème maturée), plus rarement du sirimé (beurre liquide). Elles faisaient leur commerce dans des calebasses ou des bassines à même le sol ou sur un petit étalage de fortune. Elles pouvaient vendre leur propre production ou celle revendue par des collecteurs. En fin d'après-midi, elles pouvaient se transformer en vendeuses ambulantes pour écouler les invendus.

Les boutiquiers étaient des épiciers qui proposaient de nombreux produits alimentaires et autres articles de première nécessité. Dans leurs boutiques, on trouvait systématiquement du lait en poudre et du lait concentré, parfois du lait UHT (stérilisation à ultra haute température), et certaines étaient dotées d'un réfrigérateur qui permettait de stocker des yaourts, du féné ou du lait frais. Ils s'approvisionnaient auprès des grossistes de la place pour le lait en poudre et le lait concentré, et auprès des laiteries pour les produits frais.

Les étalagistes (ou tabliers) disposaient d'un kiosque ou, plus souvent, d'une petite table pour vendre quelques produits de première nécessité (proposés généralement sous forme de microdosettes). Ils vendaient du lait en poudre en sachets de 6 à 22,5 g dans des quartiers dépourvus de boutiques à proximité immédiate.

Les cafetiers (ou cantiniers ou bars laitiers) vendaient du lait en poudre et du lait concentré pour le petit déjeuner de leurs clients. Ils se trouvaient au niveau des gares routières, des grands marchés urbains, des sorties d'administration, d'écoles ou d'usines, ou le long des principaux axes routiers.

Les transformatrices achetaient du lait frais local ou, plus souvent, du lait en poudre pour fabriquer de façon artisanale du lait caillé ou des glaces en microdosettes. Leur activité était très souple et s'adaptait à la demande saisonnière des clients urbains. Elles traitaient plusieurs dizaines à plusieurs centaines de litres par jour qu'elles commercialisaient directement ou par le biais d'un réseau de vendeuses fixes.

Les minilaiteries, qui traitaient quelques centaines à un millier de litres par jour, étaient nombreuses en zones périurbaines, notamment dans les villes secondaires.

Enfin, les laiteries, qui traitaient plusieurs milliers de litres par jour, collectaient, transformaient et distribuaient les produits laitiers. Ces produits étaient issus soit du lait local soit du lait en poudre importé.

\section{Typologie des réseaux de collecte et de distribution du lait local}

L'ordonnancement de ces acteurs et le fonctionnement de leurs réseaux suivaient deux principaux modèles discutés ci-après.

\section{Ceintures laitières de proximité}

Le modèle de la ceinture laitière est commun dans les villes soudano-sahéliennes. Le secteur périurbain bénéficie en effet des atouts offerts par la proximité d'un marché solvable. Pour autant, l'efficacité du réseau de collecte et de distribution demeure limitée. Il est ici question du poids réel des acteurs de la filière sur l'approvisionnement des villes.

Trois principales catégories d'acteurs collectaient et distribuaient du lait produit dans la périphérie des villes : les vendeuses ambulantes ou fixes, les collecteurs à vélo ou en moto et les minilaiteries. La présence sur le terrain des vendeuses et des collecteurs était relativement faible, de l'ordre de 2 à 6 pour 10000 habitants dans les villes secondaires et moins de 1 dans une capitale (tableau IV). En outre, compte tenu des modes d'approvisionnement, les quantités vendues étaient limitées (tableau V). Un cycliste et un motocycliste transportaient respectivement moins de 30 et $40 \mathrm{~L}$ par voyage. Une vendeuse ambulante se déplaçait avec 10-15 L de lait tout au plus dans sa calebasse ou son seau. Les vendeuses fixes commercialisaient à peu près le double, soit 20 à $30 \mathrm{~L}$. A titre de comparaison, les boutiquiers, beaucoup plus nombreux, commercialisaient 40 à $80 \mathrm{~L}$ équivalent lait/jour de produits à base de lait en poudre.

Augmenter les ventes serait donc synonyme d'augmentation du nombre d'acteurs du réseau ou des quantités transportées par l'amélioration de la logistique. Mais le facteur limitant reste la taille du rayon de collecte inférieur à quelques dizaines de kilomètres. Les coûts et les durées de transport deviennent vite contraignants et rédhibitoires. La situation est plus critique encore autour des capitales où la concurrence foncière avec les terres agropastorales est rude et joue invariablement en faveur de l'urbanisation. La mise en place de centres de collecte ou de minilaiteries en périphérie des villes, équipées en véhicules, permet de résoudre en partie le problème du transport vers les lieux de consommation. En revanche, le problème du rayon de collecte, qui s'appuie sur le même type d'acteurs et de moyens de transport, persiste. De ce fait, les quantités traitées par ces nouveaux opérateurs dépassent rarement $400 \mathrm{~L} /$ jour et concernent peu d'éleveurs (3). Dans ce paysage, le centre de collecte de Kassela, dans la périphérie de Bamako, fait figure d'exception en atteignant $1500 \mathrm{~L} /$ jour et en collectant à près de $50 \mathrm{~km}$ (2). Au final, les minilaiteries semblent cependant davantage être un outil performant pour rassurer des groupements de producteurs que pour assurer une augmentation sensible et durable de la couverture urbaine en produits laitiers. Ce propos est à nuancer dans certaines villes secondaires telles que Kolda ou Niono (tableau III) où elles ont effectivement pris une place prépondérante dans le paysage laitier $(7,10)$. De même, la mise en place de la laiterie Dirfel et de fermes intensives à proximité de Dakar a permis de fournir davantage de lait dans la capitale sénégalaise (6). Il n'en demeure pas moins que les quantités collectées et les producteurs concernés représentent la portion congrue d'un potentiel national sous-exploité dans tous les pays. Ce type d'organisation est sous la menace d'une expansion difficilement contrôlable des zones urbanisées qui repousse encore plus loin les zones de production potentielles.

Au-delà de ces considérations, il semble qu'un nouveau danger guette les minilaiteries. Paradoxalement, elles pourraient être victimes de leur succès. Moins de dix ans après leur mise en place, certaines se retrouvent en effet dans des situations de concurrence exacerbée sur des marchés étroits et saturés comme à Kolda au Sénégal ou Niono et Koutiala au Mali (3). Leur vulnérabilité dénote en fait leur incapacité à exporter leur production vers les marchés des grandes villes et, en premier lieu, ceux des capitales. Autrement dit, elles seraient victimes de leur enclavement commercial.

\section{Systèmes de collecte industrielle du lait}

La mise en place de réseaux de transports performants, routiers ou ferroviaires, et la mise au point de traitements technologiques, tels que la pasteurisation ou la stérilisation UHT ont profondément transformé l'organisation de l'approvisionnement en lait des grands centres urbains de part le monde (16). Autrefois cantonnés dans les ceintures laitières, les réseaux de collecte soutenus par des entreprises spécialisées et la grande distribution ont pu se 
délocaliser dans des bassins laitiers favorables à l'entretien d'animaux, à l'accès aux ressources et à la production fourragère.

Les bassins laitiers réunissent en effet un cheptel nombreux et une production potentiellement importante. Si au démarrage la collecte se heurte à l'atomisation et à la faiblesse des quantités commercialisées, les expériences industrielles soudano-sahéliennes passées (Ucolait à Saint- Louis et Nestlé à Dahra au Sénégal) et en cours (La laiterie du Berger au Sénégal, Tiviski dans la vallée du fleuve Sénégal en Mauritanie) montrent que cet écueil est rapidement surmonté $(1,3,16)$. Ainsi l'intensification de la production, notamment par l'usage raisonné d'aliments concentrés en saison sèche, et la mise en place de groupements d'éleveurs ou de coopératives associés aux centres de collecte peuvent assurer un approvisionnement conséquent toute l'année, en dépit d'une saisonnalité certaine. Le modèle choisi s'appuie, d'une part, sur des centres ruraux de collecte équipés de citernes de réfrigération et, d'autre part, sur des centres de transformation et de conditionnement localisés dans les centres urbains, au cœur des zones de consommation. Si l'entreprise s'occupe du transport entre le centre de refroidissement et la laiterie, ce sont généralement les éleveurs, au travers de leurs groupements ou de leurs coopératives qui se chargent de la collecte jusqu'aux tanks à lait. Ce système permet de limiter les coûts de transport et d'assurer la qualité sanitaire du lait. Il permet surtout de concentrer une offre laitière de plusieurs milliers de litres par jour pour la vendre dans les villes et les capitales. L'industrialisation de la collecte dans un bassin laitier apparaît par conséquent comme une solution séduisante, capable, d'une part, de sécuriser un flux laitier en qualité et en quantité et, d'autre part, de désenclaver les petits producteurs des bassins laitiers éloignés.

Mais l'optimisme est à tempérer. La privatisation du secteur de l'élevage a accompagné l'élan d'engouement pour l'industrialisation, avec des modèles variés (coopératives, entreprises). Pourtant, les expériences industrielles d'Olani (Niamey, Niger) et de MaliLait (Bamako, Mali) ont été vécues dans les années 1990 comme des

\section{Tableau IV}

Nombre d'acteurs de la filière lait pour 10000 habitants

\begin{tabular}{|c|c|c|c|c|c|c|c|}
\hline & \multirow[t]{2}{*}{ Type d'acteur } & \multicolumn{5}{|c|}{ Mali * } & \multirow{2}{*}{$\begin{array}{l}\text { Sénégal ** } \\
\text { Saint-Louis }\end{array}$} \\
\hline & & Bamako & Koutiala & Mopti & Ségou & Sikasso & \\
\hline \multirow[t]{3}{*}{ Lait local } & Collecteur & 0,3 & $?$ & 1,6 & 3,2 & 3,1 & $?$ \\
\hline & Vendeuse ambulante & 0,8 & 1,9 & 6,3 & 5,0 & $?$ & 3,0 \\
\hline & Vendeuse fixe & 0,8 & 4,3 & 47,8 & 4,9 & 5,8 & 2,8 \\
\hline \multirow[t]{3}{*}{ Lait importé } & Boutiquier & 25,3 & 26,0 & 40,1 & 34,9 & 48,5 & 20,6 \\
\hline & Cafetier & 1,8 & 11,3 & 5,2 & 6,6 & 10,8 & $?$ \\
\hline & Etalagiste & $?$ & $?$ & $?$ & 2,2 & 2,5 & $?$ \\
\hline
\end{tabular}

* D'après les enquêtes de l'étude

** D'après Corrniaux, 2005

\section{Tableau V}

Moyenne (écart-type) des quantités vendues de produits laitiers par type d'acteur et par ville (litre équivalent lait/jour)

\begin{tabular}{|c|c|c|c|c|c|c|c|}
\hline & \multirow[t]{2}{*}{ Type d'acteur } & \multicolumn{5}{|c|}{ Mali * } & \multirow{2}{*}{$\begin{array}{l}\text { Sénégal ** } \\
\text { Saint-Louis }\end{array}$} \\
\hline & & Bamako & Koutiala & Mopti & Ségou & Sikasso & \\
\hline \multirow[t]{3}{*}{ Lait local } & Collecteur & $\begin{array}{c}34,5 \\
(13,6)\end{array}$ & & $\begin{array}{c}10,8 \\
(12,6)\end{array}$ & $\begin{array}{c}37,2 \\
(18,6)\end{array}$ & & \\
\hline & Vendeuse ambulante & $\begin{array}{c}14,3 \\
(11,4)\end{array}$ & $\begin{array}{c}8,5 \\
(5,9)\end{array}$ & $\begin{array}{l}10,5 \\
(5,7)\end{array}$ & $\begin{array}{l}13,9 \\
(7,8)\end{array}$ & & \\
\hline & Vendeuse fixe & $\begin{array}{c}26,7 \\
(22,7)\end{array}$ & $\begin{array}{c}10,4 \\
(17,9)\end{array}$ & $\begin{array}{c}15,3 \\
(13,1)\end{array}$ & $\begin{array}{c}22,4 \\
(18,3)\end{array}$ & $\begin{array}{c}11,8 \\
(10,9)\end{array}$ & \\
\hline \multirow[t]{3}{*}{ Lait importé } & Boutiquier & $\begin{array}{c}49,3 \\
(67,2)\end{array}$ & $\begin{array}{c}49,3 \\
(72,7)\end{array}$ & $\begin{array}{c}77,8 \\
(44,9)\end{array}$ & $\begin{array}{c}61,3 \\
(11,4)\end{array}$ & $\begin{array}{c}56,2 \\
(21,9)\end{array}$ & $\begin{array}{c}43,0 \\
(69,9)\end{array}$ \\
\hline & Cafetier & $\begin{array}{l}10,0 \\
(5,7)\end{array}$ & $\begin{array}{c}9,9 \\
(5,7)\end{array}$ & $\begin{array}{c}12,9 \\
(22,2)\end{array}$ & $\begin{array}{c}1,2 \\
(0,7)\end{array}$ & $\begin{array}{c}6,0 \\
(4,7)\end{array}$ & \\
\hline & Etalagiste & & & & $\begin{array}{c}2,2 \\
(1,9)\end{array}$ & $\begin{array}{c}2,5 \\
(3,1)\end{array}$ & \\
\hline
\end{tabular}

* D'après les enquêtes de l'étude

** D'après Corniaux, 2005 
échecs de collecte de lait local rapidement remplacé par du lait en poudre. D'ailleurs, cette collecte provenait, et provient encore pour MaliLait comme pour Olani, de la ceinture périurbaine (limitée par la concurrence foncière) et en aucun cas d'un bassin rural de production. Finalement, aujourd'hui, Tiviski (entreprise privée qui a construit sa réussite autour du lait UHT, produit d'appel qui lui assure une marge intéressante sur un marché où les consommateurs sont friands de lait frais) en Mauritanie et La Laiterie du Berger au Sénégal sont les seules entreprises laitières qui fonctionnent sur ce modèle dans les pays soudano-sahéliens. Pour elles, la difficulté à collecter en brousse du fait de la faiblesse et de l'atomisation récurrentes de la production se traduit par un coût élevé de la collecte. En outre, les volumes demeurent bien en deçà de leurs capacités de production. Pour rentabiliser une chaîne coûteuse de pasteurisation, elles sont amenées à développer une gamme de jus de fruits, voire, dans le cas de Tiviski, à transformer du lait en poudre.

En résumé, deux types de systèmes d'approvisionnement en produits laitiers locaux permettent de satisfaire en partie les marchés urbains : la filière périurbaine qui s'appuie sur une ceinture laitière proche et la filière industrielle, centrée dans un bassin laitier de production lointain. Le modèle industriel permettrait une meilleure pénétration du marché que celui du modèle périurbain. Mais leurs difficultés à faire face à la collecte et à la demande urbaine, notamment dans les capitales, semblent au contraire favoriser la distribution du lait en poudre qui s'installe durablement (cf. discussion ci-après).

\section{DISCUSSION}

\section{Réseaux de commercialisation de la poudre}

L'Union européenne (France, Irlande), l'Amérique latine (Argentine, Brésil) et la Nouvelle-Zélande sont les principaux fournisseurs en produits laitiers de quelques dizaines d'importateurs basés dans les capitales d'Afrique subsaharienne. Ils sont quelques-uns par pays pour négocier la majorité des flux. Trois sociétés maliennes (Somakoff, Disnepal, Nima) se partageaient en valeurs 62 p. 100 du marché des produits laitiers en 2006. La Satrec *, au Sénégal, reconditionnait en volume 68 p. 100 des importations de lait en poudre en 2006. En amont, ces importateurs sont en liaison avec les sociétés laitières ou agroalimentaires internationales. En aval, ils s'appuient sur des grossistes locaux ou des laiteries qui jouent un rôle d'intermédiaires avec les détaillants. La grande distribution est peu présente. En revanche, les boutiquiers forment un réseau dense et ubiquiste dans les villes soudano-sahéliennes (cf. ci-après). Le maillage est étroit : les consommateurs ont généralement moins de 200 mètres à faire pour se rendre dans une boutique. Dans un environnement urbain où les transports sont chers, cette proximité est souvent un élément décisif dans l'achat d'un produit laitier. Les boutiquiers ont d'autres atouts. Ils proposent tous une diversité de lait en poudre et de lait concentré avec notamment un conditionnement en microdosettes adapté au mode de consommation des clients. Dans les capitales et, dans une moindre mesure, les villes secondaires, certains d'entre eux sont équipés d'un réfrigérateur qui en fait la cible privilégiée des laiteries. Enfin, à l'instar du Sénégal, des boutiquiers transforment eux-mêmes le lait en poudre pour vendre du lait caillé aux clients de leur quartier.

La filière du lait en poudre dispose d'un autre atout de poids : les laiteries basées dans les capitales travaillent quasi exclusivement sur la base de poudre de lait importée. C'est ainsi que MaliLait et Eurolait (marques Candia et Yoplait) mettent sur le marché 25000

* La Satrec (marques Vivalait, Bestlait et Roilait) a traité 17000 t sur les 25000 t importées en 2005 (Desmoulin, 2006). à 30000 L/jour à Bamako. A Dakar, les entreprises Sim, Saprolait et les Mamelles Jaboot vendent chacune au quotidien plusieurs milliers de litres de lait caillé et de yaourt alors que Kirène (marque Candia) propose du lait UHT. Particulièrement dynamiques depuis une décennie, ces laiteries multiplient les campagnes publicitaires qui témoignent à la fois de la vivacité du marché et de la réactivité des entreprises.

\section{Adaptation des réseaux urbains à la vente de produits laitiers importés}

Si elles sont des atouts indispensables, la proximité et la vente en microdosettes ne sont pas une singularité des boutiquiers, principaux pourvoyeurs des produits laitiers importés. Les vendeuses ambulantes garantissent aussi la proximité en faisant du porte à porte et elles vendent leurs laits produits localement par petites louches. Mais une caractéristique fondamentale différencie ces deux réseaux de distribution : leur présence effective sur le terrain urbain et leur capacité de vente. En ville on dénombre plusieurs dizaines de boutiques pour 10000 habitants alors que le ratio est de l'ordre de quelques unités pour les vendeuses ambulantes. Elles sont moins d'une pour 10000 habitants à Dakar et à Bamako (tableau IV). De plus, un boutiquier peut distribuer plusieurs dizaines de litres de lait par jour quand la vendeuse ambulante ne peut dépasser $15 \mathrm{~L}$ (tableau V). Finalement, la combinaison des deux facteurs conduit à un rapport de force de distribution de 30 à 80 fois supérieur chez les boutiquiers face à celui des vendeuses ambulantes, principaux détaillants respectivement pour le lait importé et le lait local.

Le niveau le moins élevé de ce rapport est observé dans les villes secondaires. Même s'il faut nuancer la comparaison en considérant que les ventes des boutiquiers sont aussi destinées à des consommateurs ruraux (les villages de la région s'approvisionnent en ville), la différence de «puissance » entre les deux réseaux demeure respectable. Le rôle du facteur prix, jugé comme déterminant dans l'acte de choix entre lait importé et lait local, peut dès lors être relativisé. Ni la dévaluation du franc CFA, ni l'enclavement d'une capitale, ni l'augmentation du cours mondial du lait en poudre en 2007 (prix doublé), ni la proximité d'un lait concurrentiel local (dans les villes secondaires) n'influe durablement sur un éventuel infléchissement des ventes de lait importé par les boutiquiers. A titre d'exemple, la dévaluation de 50 p. 100 du franc CFA en 1994 a un temps affecté les importations de lait en poudre mais n'a pas engendré un développement spectaculaire de la filière locale. Le même constat est à faire lors du doublement du prix de la poudre sur le marché mondial courant 2007. Le grand écart actuel s'explique par la faculté des importateurs et des boutiquiers à répondre rapidement à des variations de demandes, en quantité et en qualité, c'est-à-dire justement ce à quoi ne peuvent s'adapter à ce jour les acteurs de la filière locale.

\section{Appuyer la collecte : un effort nécessaire et suffisant?}

Afin d'assurer une meilleure couverture des besoins urbains en lait, il apparaît désormais qu'un effort sur le réseau de collecte soit nécessaire. Pour autant, est-il suffisant? Les auteurs viennent de montrer que la concurrence des réseaux de distribution de la poudre en limiterait les effets, même en cas de différentiels de prix en faveur du lait local. Mais les contraintes ne s'arrêtent pas à ce niveau. Les obstacles demeurent importants aussi bien en amont qu'en aval : existe-t-il une production commercialisable effective et surtout pour quel marché ?

A la première question, les autorités publiques proposent depuis plusieurs décennies des solutions techniques (insémination artificielle, complémentation alimentaire) qui ont montré leurs limites 
$(4,16,17)$. Les raisons de la stagnation et de la faiblesse de la production locale sont désormais à chercher ailleurs. Elles relèvent sans doute davantage des facultés de la profession à considérer la production laitière comme une priorité dans leurs systèmes d'activités. Autrement dit, il s'agit de passer d'un statut d'éleveur ou d'agroéleveur à celui de producteur laitier à part entière, dans un environnement à risques. Les systèmes de collecte ont ici leur rôle à jouer en donnant des garanties à leurs fournisseurs, notamment via la contractualisation. Mais ne fragilise-t-on pas alors les exploitations en limitant leur stratégie de diversification?

A la seconde question, les réponses sont moins tranchées. Certes, il apparaît que les habitudes alimentaires urbaines se modifient rapidement et plutôt en faveur du lait en poudre (10). Cependant, les études actualisées sont rares et partielles. On possède trop peu d'éléments sur la dynamique de la consommation des produits laitiers et de ses facteurs (prix, goût, santé...). Ils sont pourtant déterminants dans l'approche du développement de la filière locale.

\section{CONCLUSION}

En Afrique soudano-sahélienne, la faiblesse du réseau de collecte et de distribution du lait local apparaît comme un sérieux frein au développement du secteur laitier. A contrario, les entreprises, qui transforment le lait en poudre, et les boutiquiers, solides points de vente des produits laitiers importés, captent les bénéfices de l'élargissement du marché laitier consécutif à l'augmentation de la population urbaine. L'évolution des habitudes alimentaires joue d'ailleurs en leur faveur. Aujourd'hui, les acteurs de la collecte et de la distribution du lait local ne peuvent répondre à la demande urbaine en lait que de façon partielle. Dans les villes secondaires, la proximité des lieux de production, la présence effective de ces acteurs sur le terrain et la mise en place de minilaiteries de statut privé ou coopératif sont des atouts indéniables qui garantissent une part du marché laitier. En revanche, dans les capitales, les lieux de production périurbains, bien qu'intensifiés, sont repoussés de plus en plus loin rendant la collecte traditionnelle hasardeuse. A défaut de développement d'une collecte industrielle adossée à un bassin laitier de production, les grandes villes offrent ainsi leur marché laitier aux réseaux organisés et performants du lait en poudre.

Au fond, la faiblesse du circuit local réside d'abord dans la dispersion de ses acteurs et dans la faiblesse des flux mis sur le marché, rejoignant ici les conclusions de Thomas et coll. (15) qui donnaient l'image suivante pour caractériser la situation du secteur malien de la distribution du lait local : « on pourrait comparer ces deux pôles que sont la production et la consommation à deux grands lacs, un lac d'amont et un lac d'aval, plutôt distants l'un de l'autre et actuellement reliés par un réseau diffus de petits canaux traversant une zone ensablée. Le flux est faible entre les deux réservoirs, et le réservoir d'aval à recours à des sources extérieures (importations) pour combler le déficit, pendant que le réservoir d'amont ne trouve pas d'exutoire et stagne ». Pour garder cette image, on peut dire qu'avec l'urbanisation la situation dans les villes secondaires est sans doute en cours d'ensablement, en dépit de la mise en place de quelques minilaiteries dont le débit reste trop modeste pour infléchir la tendance.

La taxation des produits laitiers importés apparaît trop souvent à travers des discours partisans et médiatisés $(12,14)$ comme la principale mesure à prendre en faveur du sauvetage de la filière locale. Il s'agit de jouer sur le facteur prix pour rendre plus compétitif le lait produit localement. Mais cette « discrimination positive » n'est à l'évidence pas suffisante. La récente augmentation du prix mondial du lait en poudre (2007) et la dévaluation du franc CFA de 1994 prouvent que d'autres facteurs entrent en jeu, comme l'accessibilité des produits locaux. S'il existe une réelle volonté de promouvoir la production locale, l'appui à la collecte et à la mise en place d'infrastructures de refroidissement et de stockage devrait par conséquent être considéré par les responsables de la politique laitière en Afrique soudano-sahélienne comme prioritaire. Des actions incitatives pourraient par exemple être proposées par l'Etat en direction des structures privées au travers d'exonérations ou de l'instauration de quotas négociés avec les entreprises présentes. A terme, la distribution pourrait même s'appuyer sur le réseau des boutiquiers disposant de réfrigérateurs. Mais la collecte ne pourra être efficace que si les producteurs adhèrent au projet. Cela passe par la lutte contre l'atomisation de la production et contre un comportement individualiste (en particulier dans la captation des marges). Or l'esprit coopératif est encore déficient dans le milieu pastoral. Au fond, n'est-ce pas là la principale faiblesse actuelle de la filière locale face aux importations de poudre de lait ?

\section{Remerciements}

Le présent article est issu de travaux financés par le projet Coraf Politiques laitières et le Pôle suisse de compétence dans la recherche (Nccr) Nord-Sud : Partenariat de recherche pour l'atténuation des syndromes du changement global. Nous adressons également nos remerciements aux relecteurs qui ont permis d'améliorer sensiblement cet article.

\section{BIBLIOGRAPHIE}

1. ABEIDERRAHMANE N., 2001. Collecte, transformation et commercialisation du lait pour I'approvisionnement de Nouakchott : I'expérience de la Laitière de Mauritanie. In : Duteurtre G., Meyer G., éds, Marchés urbains et développement laitier en Afrique subsaharienne. Montpellier, France, Cirad, 233 p.

2. BONFOH B., ANKERS P., SALL A., DIABATE M., TEMBELY S., FARAH Z., ALFAROUKH I.O., ZINSSTAG J., 2005. Schéma fonctionnel de services aux petits producteurs laitiers périurbains de Bamako (Mali). Etud. Rech. sahél. (12) : 7-25.

3. CORNIAUX C., 2003. La filière lait et les produits laitiers dans la région de St Louis. Rapport de synthèse. Montpellier, France, Cirad, 52 p. + annexes.
4. CORNIAUX C., 2005. Gestion technique et gestion sociale de la production laitière : les champs du possible pour une commercialisation durable du lait - Cas des systèmes d'élevage actuels du delta du fleuve Sénégal. Thèse Doct. INA-PG, Paris, France, 242 p. + annexes.

5. CORNIAUX C., DUTEURTRE G., DIEYE P.N., POCCARD-CHAPUIS R. 2005. Les minilaiteries comme modèle d'organisation des filières laitières en Afrique de l'Ouest : succès et limites. Revue Elev. Méd. vét. Pays trop., 58 : 237-243.

6. DESMOULIN X., 2006. L'approvisionnement de Dakar en produits laitiers états des lieux et perspectives. Mém. Master, Université Paris XI, France, 184 p.

7. DIEYE P.N., 2006. Arrangements contractuels et performances des marchés du lait local au sud du Sénégal. Les petites entreprises face aux incertitudes de I'approvisionnement. Thèse Doct. Ensa, Montpellier France, 175 p. + annexes. 
8. DIEYE P.N., FAYE A., SEYDI M., CISSE S.A., 2002. Production laitière périurbaine et amélioration des revenus des petits producteurs en milieu rural au Sénégal. Cah. Agric., $11: 251-257$.

9. DUTEURTRE G., 2000. L'organisation de la filière laitière autour de Niono. Rapport de mission. Montpellier, France, Cirad, 31 p.

10. DUTEURTRE G., CORNIAUX C., BOUTONNET J.P., 2003. Baisse de la consommation des produits laitiers en Afrique subsaharienne : mythe ou réalité ? Renc. Rech. Ruminants, 10 : 323-326.

11. METZGER R., CENTRES J.M., THOMAS L., LAMBERT J.C., 1995. L'approvisionnement des villes africaines en lait et produits laitiers. Rome, Italie, FAO, 105 p. (Prod. Santé anim. $\mathrm{n}^{\circ}$ 124)

12. OXFAM, 2002. La PAC pressurée. Comment le régime laitier de I'Europe est en train de ruiner les moyens d'existence du monde en développement. Paris, France, Oxfam, 39 p. (Document $n^{\circ} 34$ )

13. POCCARD-CHAPUIS R., CORNIAUX C., 2007. La demande urbaine en produits laitiers : contrastes entre la capitale et les villes secondaires du Mali. Renc. Rech. Ruminants, 14: 454.

\section{Summary}

Corniaux C., Bonfoh B., Diallo A., Poccard-Chapuis R., Vias G. Milk Collection and Distribution Networks in African Sudano-Sahelian Cities

In secondary African Sudano-Sahelian cities, proximity to rural areas still allows milk collection from producers in periurban zones and its distribution to urban consumers. Many actors transport and trade small quantities. Small dairy farms sometimes play a major role as intermediaries. On the other hand, the gap is widening in capitals as the collection and distribution network is not adapting to market needs. Although small dairy farms are dynamic, they only bring partial answers to the need to adapt. The population growth, the urban expansion at the periphery of cities to the detriment of pastures or the increasing distance between production and consumption make obsolete the traditional system of transportation. For milk production, to keep a significant role in the potential urban market, "industrial" collection in production zones should be promoted in spite of the distance as in the case of Tiviski in Mauritania. But industrialization conditions are not easily met. In addition, the distribution of imported powder milk and its derived products respond efficiently to recent transformations in urban markets (multiplication of shops, product diversification, increase of cold chains, attractive prices, good sanitary quality...) making competitiveness harder or even elusive. Public policy should take into account this situation. Supporting local milk collection and distribution is a priority with the reservation that local production development must also be a priority of decision makers.

Keywords: Milk collection - Marketing channel - Network analysis - Urban environment - Africa.
14. SOS FAIM, 2006. De la vache au lait, du lait à la poudre de lait, de I'Europe à l'Afrique : une production et une consommation, qui ne se rencontrent pas toujours. Dyn. Paysannes (13) : 1-12.

15. THOMAS L., LE MASSON A., DIAKITE N., BRUART J., 1996. Appui à la formulation d'un programme national de promotion de la production laitière au Mali. Rome, Italie, FAO, 158 p. + annexes.

16. VATIN F., 1996. Le lait et la raison marchande. Essais de sociologie économique. Rennes, France, Presses universitaires de Rennes, 205 p.

17. VATIN F., 1992. L'industrialisation laitière impossible. Réflexions sur le cas de I'Afrique soudano-sahélienne. In : Corbel P., Vatin F., dir., Mondes ruraux en mutation. Rennes, France, Presses universitaires de Rennes, 13 p.

Accepté le 01.10.2009

\section{Resumen}

Corniaux C., Bonfoh B., Diallo A., Poccard-Chapuis R., Vias G. Sistemas de colecta y de distribución de leche en las ciudades de Africa sudano sahelina

En las ciudades secundarias de Africa sudano sahelina, la proximidad del medio rural permite aún la colecta de leche directamente de los productores situados en zona peri urbana y la distribución de esta directamente a los consumidores urbanos. Numerosas entidades transportan y comercializan pequeñas cantidades. Las mini lecherías ocupan a veces un lugar mayor como relevo. Por otro lado, se está creando una brecha en las capitales, la cuál continuará en cuanto la red de colecta y de distribución no se adapte a las exigencias del mercado. Las mini lecherías, a pesar de su dinamismo, responden únicamente en forma parcial a esta necesidad de adaptación. El crecimiento demográfico, la expansión urbana hacia la periferia en detrimento de los pastizales o bien la lejanía progresiva de la producción con respecto a los lugares de consumo hacen caducos los sistemas de transporte. Para que la producción lechera local mantenga un lugar significativo en el mercado urbano rentable, la colecta "industrial" en los lugares de producción debería de ser promovida a pesar de la lejanía, como ocurre en la lechería Tibiski en Mauritania. Pero las condiciones para la industrialización no son fáciles de reunir. Además, las redes de distribución de leche en polvo importado y de sus productos derivados siguen eficazmente las transformaciones recientes de los mercados urbanos (multiplicaciones de las tiendas, diversificación de los productos, presencia creciente de la cadena de frío, precios atractivos, calidad sanitaria correcta...), dificultan todavía más, de forma casi ilusoria, la competencia. Las políticas públicas deben tomar en cuenta este hecho. El apoyo de las unidades de colecta y de distribución de leche local es una prioridad, bajo reserva de que el desarrollo de la producción local sea válido a los ojos de los dirigentes.

Palabras clave: Recogida de leche - Corriente de mercadeo Análisis de redes - Ambiente urbano - Africa. 